\title{
Optimal Control for Transmission of Water Pollutants
}

\author{
Nita H. Shah ${ }^{*}$, Shreya N. Patel², Moksha H. Satia ${ }^{3}$, Foram A. Thakkar \\ Department of Mathematics \\ Gujarat University, Ahmedabad-380009, Gujarat, India \\ E-mails: ${ }^{* 1}$ nitahshah@gmail.com, ${ }^{2}$ patelshreya513@gmail.com, ${ }^{3}$ mokshastia.05@gmail.com, \\ ${ }^{4}$ foramkhakhar3@gmail.com \\ ${ }^{*}$ Corresponding author
}

(Received February 22, 2018; Accepted April 7, 2018)

\begin{abstract}
Pollutants are formed when oil, gas, chemical plants, etc. discharge their harmful waste materials into stream or other water bodies. In this paper, a mathematical model for water pollutants which are soluble and insoluble has been formulated as a system of non-linear ordinary differential equations. Control is applied on insoluble water pollutants to process them into soluble water pollutants. Numerical simulation has been carried out which suggest that soluble water pollutants are increasing as compared to insoluble water pollutants.
\end{abstract}

Keywords- Water pollutants, Mathematical model, Basic reproduction number, Stability, Control.

\section{Introduction}

Water pollution is the burning issue for the environment. It is caused when water pollutants are discharged into water bodies like lakes, streams, oceans, etc. If they are discharged directly or indirectly without any treatment they become more harmful. Water pollution is also caused by oil refineries, construction sites, chemical wastes, some radioactive wastes, etc. They are harmful to all aquatic animals, environment and human.

Water pollutants can be classified into two categories soluble water pollutants and insoluble water pollutants. The pollutants which can dissolve in water directly without any treatment are called soluble water pollutants. As for example soap, nitrates, chlorates, acetates, all hydroxides and many more. On other hand water pollutants which do not dissolve in water without treatment are called insoluble water pollutants. For example, oil, plastic, chemicals from industries, etc. Some chemicals which are insoluble in water can be made solute with proper treatment.

The mathematical model helps to study the transmission of several issues not only in infectious diseases but also in medicine, biology, social science, also to study the spread of different kind of pollutants etc. which is cited by Ferguson et al. (2006). A model was formulated with three differential equations by Anderson (1991), Hethcote (1994) and Brauer (2008).

In this paper, a mathematical model is formulated for transmission of water pollutants in section 2. The stability analysis of the transmission model is derived in section 3. Sensitivity analysis is carried out in section 4. Optimal control for the water pollutants is discussed in section 5. In section 6 , the model is validated with numerical simulation and analysis. 
International Journal of Mathematical, Engineering and Management Sciences

Vol. 3, No. 4, 381-391, 2018

https://dx.doi.org/10.33889/IJMEMS.2018.3.4-027

\section{Mathematical Model}

Water is an essential part of the living body. Therefore, the proposed model is divided into three discrete compartments: the cubic volume of water pollutants $\left(W_{P}\right)$, the cubic volume of soluble water pollutants $(S)$ and the cubic volume of insoluble water pollutants $(I)$ are considered. Here, $u_{1}$ is the control rate for the treatment of insoluble water pollutants. We formulate a mathematical model for the analysis of solubility and insolubility of water pollutants. The notations and parametric values for the mathematical model are exhibited in Table 1.

Table 1. Notations and its parametric values

\begin{tabular}{|c|l|c|}
\hline Notations & \multicolumn{1}{|c|}{ Description } & Parametric values \\
\hline$B$ & Water pollutants rate & 0.7 \\
\hline$\beta_{1}$ & The rate of water pollutants which are soluble & 0.18 \\
\hline$\beta_{2}$ & The rate of water pollutants which are insoluble & 0.02 \\
\hline$\delta$ & The rate at which insoluble water pollutants becomes solute after treatment & 0.3 \\
\hline$\varepsilon$ & The rate of insoluble water pollutants resulting water pollution & 0.1 \\
\hline$\mu$ & The removable rate of water pollutants of each compartment & 0.4 \\
\hline$u_{1}$ & Control rate for the treatment of insoluble water pollutants & {$[0,1]$} \\
\hline
\end{tabular}

Using notations given in Table 1 transmission of water pollutants can be described as shown in Fig. 1.

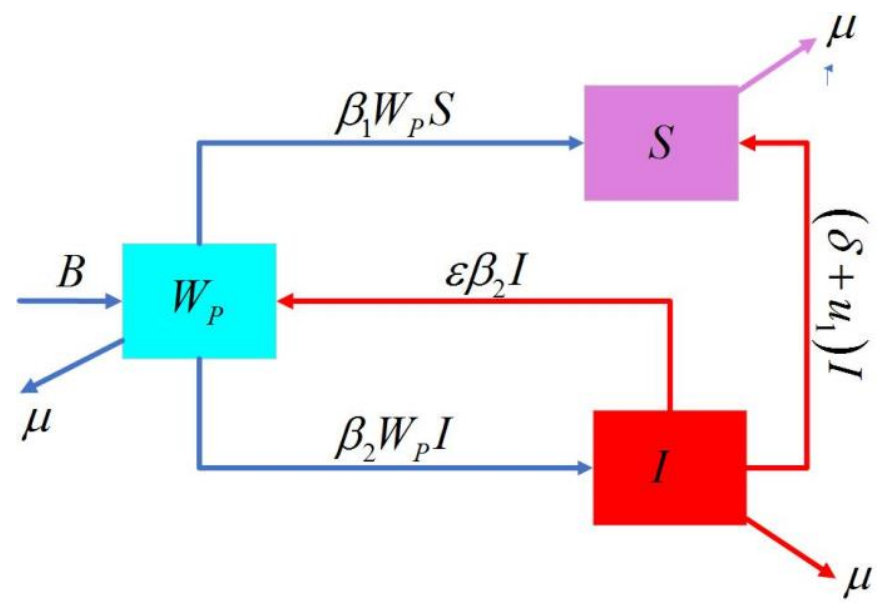

Fig. 1. Transmission of water pollutants 
International Journal of Mathematical, Engineering and Management Sciences

Vol. 3, No. 4, 381-391, 2018

https://dx.doi.org/10.33889/IJMEMS.2018.3.4-027

The governing differential equations are

$$
\begin{aligned}
& \frac{d W_{P}}{d t}=B-\beta_{1} W_{P} S-\beta_{2} W_{P} I+\varepsilon \beta_{2} I-\mu W_{P} \\
& \frac{d S}{d t}=\beta_{1} W_{P} S+\left(\delta+u_{1}\right) I-\mu S \\
& \frac{d I}{d t}=\beta_{2} W_{P} I-\left(\delta+u_{1}\right) I-\varepsilon \beta_{2} I-\mu I
\end{aligned}
$$

with $W_{P}+S+I \leq V$ and $W_{P}>0, S, I \geq 0$.

Adding all the above equations, we get

$\frac{d}{d t}\left(W_{P}+S+I\right)=B-\mu\left(W_{P}+S+I\right) \geq 0$

This gives $\limsup _{t \rightarrow \infty}\left(W_{P}+S+I\right) \leq \frac{B}{\mu}$.

Therefore, the feasible region for the system (1) is

$$
A=\left\{\left(W_{P}+S+I\right): W_{P}+S+I \leq \frac{B}{\mu}, W_{P}>0 ; S>o, I>0\right\} .
$$

Thus, the equilibrium point $E_{0}=\left(\frac{B}{\mu}, 0,0\right)$.

Next, the basic reproduction number $R_{0}$ can be calculated using the next generation matrix method of Diekmann et al. (2009).

Let $\mathrm{X}=\left(S, I, W_{p}\right)$

thus, $\frac{d X}{d t}=F(X)-V(X)$

where, $F(X)=\left[\begin{array}{c}\beta_{1} W_{P} S \\ \beta_{2} W_{P} I \\ 0\end{array}\right]$ and $V(X)=\left[\begin{array}{c}\mu S-\left(\delta+u_{1}\right) I \\ \mu I+\left(\delta+u_{1}\right) I+\varepsilon \beta_{2} I \\ -B+\beta_{1} W_{P} S+\beta_{2} W_{P} I-\varepsilon \beta_{2} I+\mu W_{P}\end{array}\right]$.

Now, the derivative of $\mathrm{F}$ and $\mathrm{V}$ of order $3 \times 3$ defined as

$$
f=\left[\frac{\partial F_{i}\left(E_{0}\right)}{\partial X_{j}}\right], v=\left[\frac{\partial V_{i}\left(E_{0}\right)}{\partial X_{j}}\right] \text { for } i, j=1,2,3
$$


International Journal of Mathematical, Engineering and Management Sciences

Vol. 3, No. 4, 381-391, 2018

https://dx.doi.org/10.33889/IJMEMS.2018.3.4-027

where, $f=\left[\begin{array}{ccc}\frac{\beta_{1} B}{\mu} & 0 & 0 \\ 0 & \frac{\beta_{2} B}{\mu} & 0 \\ 0 & 0 & 0\end{array}\right]$ and $\quad v=\left[\begin{array}{ccc}\mu & -\left(\delta+u_{1}\right) & 0 \\ 0 & \mu+\left(\delta+u_{1}\right)+\varepsilon \beta_{2} & 0 \\ \frac{\beta_{1} B}{\mu} & \frac{\beta_{2} B}{\mu}-\varepsilon \beta_{2} & \mu\end{array}\right]$.

Now, the basic reproduction number $R_{0}$ is the spectral radius of the matrix $f v^{-1}$ where

$$
R_{0}=\frac{B\left(\mu \beta_{1}+\beta_{1} \delta+\beta_{1} u_{1}+\beta_{1} \varepsilon \beta_{2}+\mu \beta_{2}\right)}{\mu^{2}\left(\mu+\delta+u_{1}+\varepsilon \beta_{2}\right)}
$$

In next section, equilibrium of the water pollutants transmission model is discussed.

\section{Stability of Equilibrium}

The equilibrium for the local and global stability of the water pollutants model is deliberated here.

\subsection{Local Stability}

The water pollution resources equilibrium is locally asymptotically stable if all the eigenvalues of the matrix have positive real values. The Jacobian matrix for the system at $E_{0}=\left(\frac{B}{\mu}, 0,0\right)$ is given by

$$
J=\left[\begin{array}{ccc}
-\mu & \frac{-\beta_{1} B}{\mu} & \frac{-\beta_{2} B}{\mu}+\varepsilon \beta_{2} \\
0 & \frac{\beta_{1} B}{\mu}-\mu & \delta+u_{1} \\
0 & 0 & \frac{\beta_{2} B}{\mu}-\left(\delta+u_{1}\right)-\mu-\varepsilon \beta_{2}
\end{array}\right] .
$$

Thus, trace $(J)<0$ provided $\min \left\{\frac{\beta_{1} B}{\mu^{2}}, \frac{\beta_{2} B}{\mu\left[\left(\delta+u_{1}\right)+\mu+\varepsilon \beta_{2}\right]}\right\}<1$.

Hence, the system (1) is locally stable.

On solving the set of equation (1), we get the other two points namely, $E_{1}=\left(\frac{\mu}{\beta_{1}}, \frac{B \beta_{1}-\mu^{2}}{\mu \beta_{1}}, 0\right)$ means insoluble water pollutants does not exist and $E^{*}=\left(W_{P}{ }^{*}, S^{*}, I^{*}\right)$ when all kind of pollutants exists. 
where,

$$
\begin{aligned}
W_{P}^{*} & =\frac{\delta+\varepsilon \beta_{2}+\mu}{\beta_{2}}, \\
S^{*} & =\frac{B \delta \beta_{2}-\mu \delta^{2}-\mu \delta \varepsilon \beta_{2}-\mu^{2} \delta}{\mu\left(-\mu \beta_{1}+\mu \beta_{1}-\beta_{2} \delta+\delta \beta_{1}+\beta_{1} \varepsilon \beta_{2}\right)}, \\
I^{*} & =\frac{\left(\delta \beta_{1}+\beta_{1} \varepsilon \beta_{2}+\mu \beta_{1}-\mu \beta_{1}\right)\left(-\mu^{2}-\mu \delta+B \beta_{2}-\mu \varepsilon \beta_{2}\right)}{\mu \beta_{2}\left(-\mu \beta_{2}+\mu \beta_{1}-\beta_{2} \delta+\delta \beta_{1}+\beta_{1} \varepsilon \beta_{2}\right)} .
\end{aligned}
$$

\subsection{Global Stability}

The water pollutants transmission model is globally stable (Shah et al., 2016, 2017) if

$\operatorname{det}\left(1-f v^{-1}\right)>0$.

$\operatorname{det}\left(1-f v^{-1}\right)=1-R_{0}=1-0.8373=0.1627>0$

therefore, the system (1) is also globally stable.

\section{Sensitivity Analysis}

In this section, the sensitivity analysis for all parameters is discussed in Table 2 . The normalised sensitivity index of the parameters is computed by using the following formula $\gamma_{\alpha}^{R_{0}}=\frac{\partial R_{0}}{\partial \alpha} \cdot \frac{\alpha}{R_{0}}$ where $\alpha$ denotes the model parameter

Table 2. Sensitivity analysis

\begin{tabular}{|c|c|}
\hline Parameter & Value \\
\hline$B$ & + \\
\hline$\beta_{1}$ & + \\
\hline$\beta_{2}$ & + \\
\hline$\delta$ & - \\
\hline$\varepsilon$ & - \\
\hline$\mu$ & - \\
\hline
\end{tabular}

The recruitment rate $(B)$, the rate of water pollutants which are soluble $\left(\beta_{1}\right)$, the rate of water pollutants which are insoluble $\left(\beta_{2}\right)$ have positive effect which means they are helping us to know water pollutants. Other parameters have negative impact on the model.

\section{Optimal Control Model}

The objective of the model is to minimize the number of insoluble water pollutants. The control function is united to achieve the objective. The objective function for the mathematical model of water pollutants in the system (1) along with the optimal control is given by 
$J\left(u_{i}, \Omega\right)=\int_{0}^{T}\left(A_{1} W_{P}^{2}+A_{2} S^{2}+A_{3} I^{2}+w_{1} u_{1}^{2}\right) d t$

where, $\Omega$ denotes set of all compartmental variables, $A_{1}, A_{2}, A_{3}$ denote non-negative weight constants for $W_{P}, S, I$ compartment respectively. As, the weight parameters $w_{1}$ is constant for control rate on in solute water pollutants to give treatment $\left(u_{1}\right)$ from which the optimal control condition is normalized. $u_{1}$ is the control rate which minimizes the insoluble water pollutants. To complete the value of control variable $u_{1}$ from $t=0$ to $t=T$ such that

$J\left(u_{1}(t)\right)=\min \left\{J\left(u_{i}^{*}, \Omega\right) / u_{1} \in \phi\right\}$

where, $\phi$ is smooth function on the interval $[0,1]$. The optimal denoted by $u_{i}^{*}, i=1$ are found by accumulation all the integrands of equation (3) using the lower bounds and upper bounds respectively with the results of Fleming and Rishel (2012).

Now, using the Pontrygin's (1986) to minimize the cost function in (3) by constructing Lagrangian function consisting of state equations and adjoint variables $A_{i}=\left(\lambda_{1}, \lambda_{2}, \lambda_{3}\right)$ as

$$
\begin{aligned}
L\left(\Omega, A_{i}\right)= & A_{1} W_{p}^{2}+A_{2} S^{2}+A_{3} I^{2}+w_{1} u_{1}^{2}+\lambda_{1}\left(B-\beta_{1} W_{P} S-\beta_{2} W_{P} I+\varepsilon \beta_{2} I-\mu W_{P}\right) \\
& +\lambda_{2}\left(\beta_{1} W_{P} S+\delta I+u_{1} I-\mu S\right)+\lambda_{3}\left(\beta_{2} W_{P} I-\delta I-u_{1} I-\varepsilon \beta_{2} I-\mu I\right)
\end{aligned}
$$

The partial derivative of the Lagrangian function with respect to each variable of the compartment gives the adjoint equation variable $A_{i}=\left(\lambda_{1}, \lambda_{2}, \lambda_{3}\right)$ corresponding to the system (1) which as follows:

$$
\begin{aligned}
& \dot{\lambda}_{1}=-\frac{\partial L}{\partial W_{P}}=-2 A_{1} W_{P}+\left(\lambda_{1}-\lambda_{2}\right) \beta_{1} S+\left(\lambda_{1}-\lambda_{3}\right) \beta_{2} I+\lambda_{1} \mu \\
& \dot{\lambda}_{2}=-\frac{\partial L}{\partial S}=-2 A_{2} S+\left(\lambda_{1}-\lambda_{2}\right) \beta_{1} W_{P}+\lambda_{2} \mu \\
& \dot{\lambda}_{3}=-\frac{\partial L}{\partial I}=-2 A_{3} I+\left(\lambda_{1}-\lambda_{3}\right) \beta_{2} W_{P}+\left(\lambda_{3}-\lambda_{1}\right) \varepsilon \beta_{2}+\left(\lambda_{3}-\lambda_{2}\right) \delta+\left(\lambda_{3}-\lambda_{2}\right) u_{1}+\lambda_{3} \mu
\end{aligned}
$$

The necessary condition for Lagrangian function $L$ to be optimal for control is

$$
\dot{u}_{1}=-\frac{\partial L}{\partial u_{1}}=-2 w_{1} u_{1}+\left(\lambda_{3}-\lambda_{2}\right) I
$$

To find the value $u_{1}$ of solving the equation (8) then

$$
u_{1}=\frac{I\left(\lambda_{3}-\lambda_{2}\right)}{2 w_{1}}
$$


International Journal of Mathematical, Engineering and Management Sciences

Vol. 3, No. 4, 381-391, 2018

https://dx.doi.org/10.33889/IJMEMS.2018.3.4-027

Thus, the required optimal control condition is computed as

$u_{1}^{*}=\max \left(a_{1}, \min \left(b_{1}, \frac{\left(\lambda_{3}-\lambda_{2}\right) I}{2 w_{1}}\right)\right)$

In next section, the optimal control is calculated numerically to support the analytical result for insoluble water pollutants model.

\section{Numerical Simulation}

Using the data given in Table 1 and Table 2, the sensitivity on model parameters is carried out.

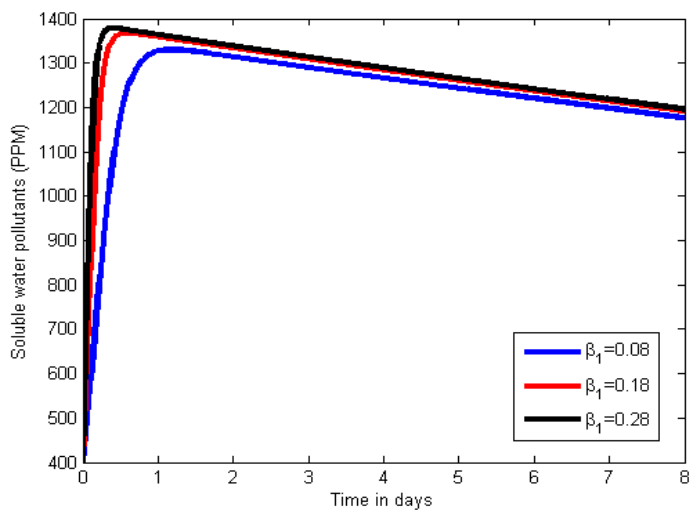

Fig. 2. Effect for the rate of water pollutants which are soluble

Fig. 2 indicates that if we increase the rate of water pollutants, which are soluble $\left(\beta_{1}\right)$ from $8 \%$ to $28 \%$, the soluble water pollutants increase in beginning then after it decreases at a slower rate.

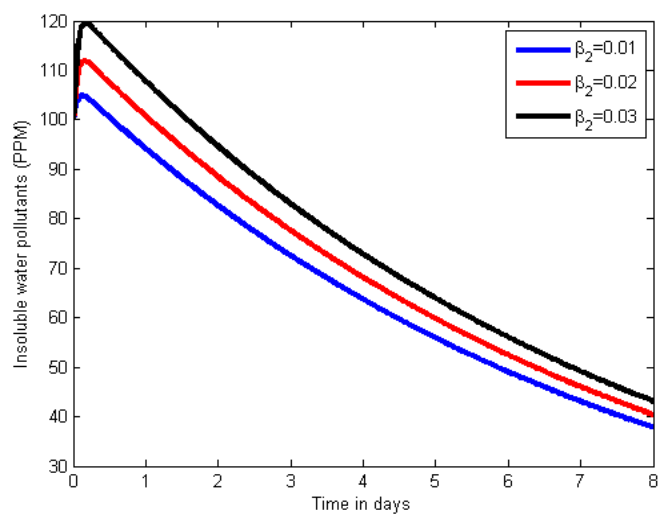

Fig. 3. Effect for the rate of water pollutants which are insoluble 
International Journal of Mathematical, Engineering and Management Sciences

Vol. 3, No. 4, 381-391, 2018

https://dx.doi.org/10.33889/IJMEMS.2018.3.4-027

From Fig. 3, one can specify that if the rate of water pollutants, which are insoluble $\left(\beta_{2}\right)$ is increased from $1 \%$ to $3 \%$, then insoluble water pollutants increases approximately by 104 PPM to 120 PPM.

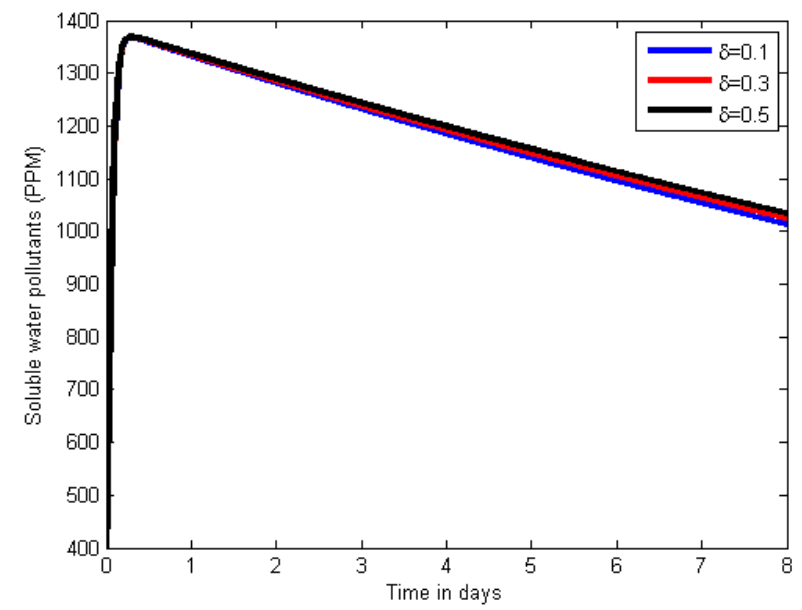

Fig. 4. Effect for the rate of insoluble water pollutants becomes solute after some treatment

Fig. 4 shows that if one will increase the rate at which insoluble water pollutants becomes solute after some treatment $(\delta)$, then the soluble water pollutants decreases only by $2 \%$ with the time. But the reduction of solute is comparatively less which indicates that government should apply control on solute water pollutants.

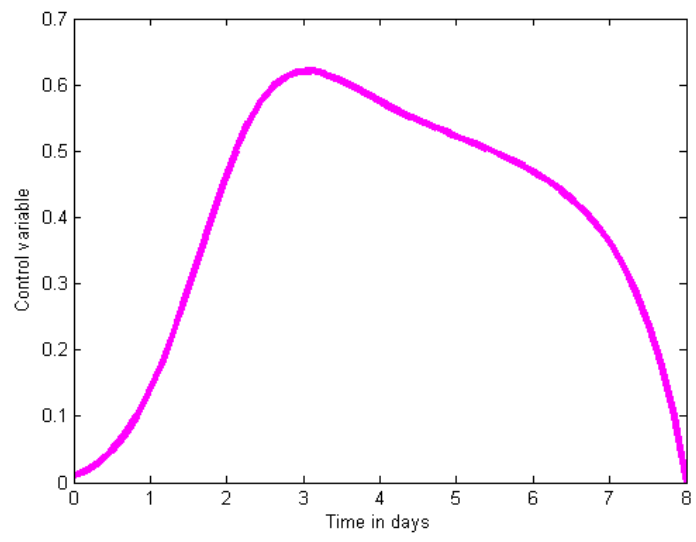

Fig. 5. Control variable verses time (in days) 
From Fig. 5, one can observe that insoluble water pollutants should be controlled $62 \%$ in almost 3 days and then it can be decreased it treatment is extended for 5 days more.

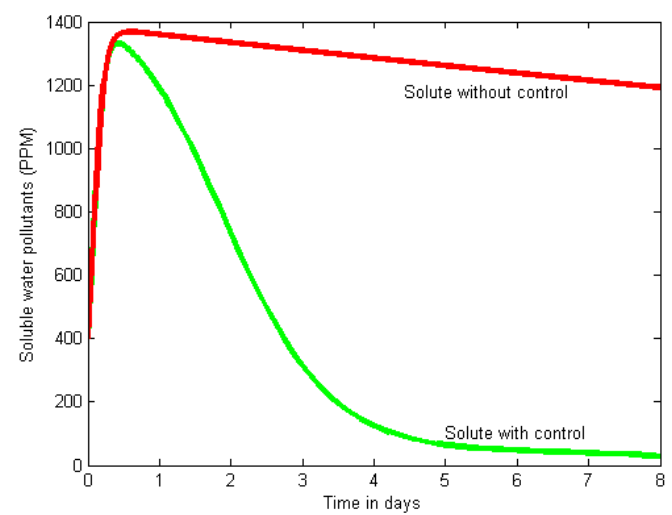

Fig. 6(a). Soluble water pollutants with and without control

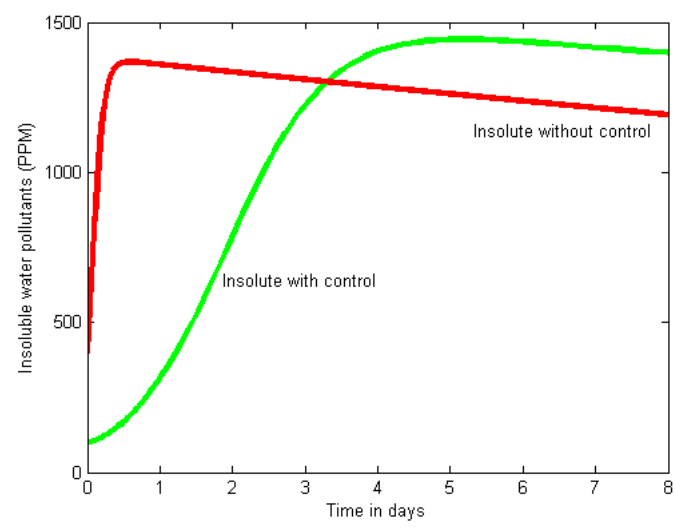

Fig. 6(b). Insoluble water pollutants with and without control

Fig. 6(a) and Fig. 6(b) indicates that on making use of chemical reaction soluble water pollutant decreases exponentially and insoluble chemicals are increasing with control at a lower rate as compared to when no control is applied. This suggests that control in terms of some treatment is beneficial to revive water pollution.

The parametric values used for following three figures are:

$B=0.7, \beta_{1}=0.15, \beta_{2}=0.6, \varepsilon=0.1, \delta=0.3, \mu=0.29$ that defines the equilibrium point $E_{0}, E_{1}$ and $E^{*}$ numerically

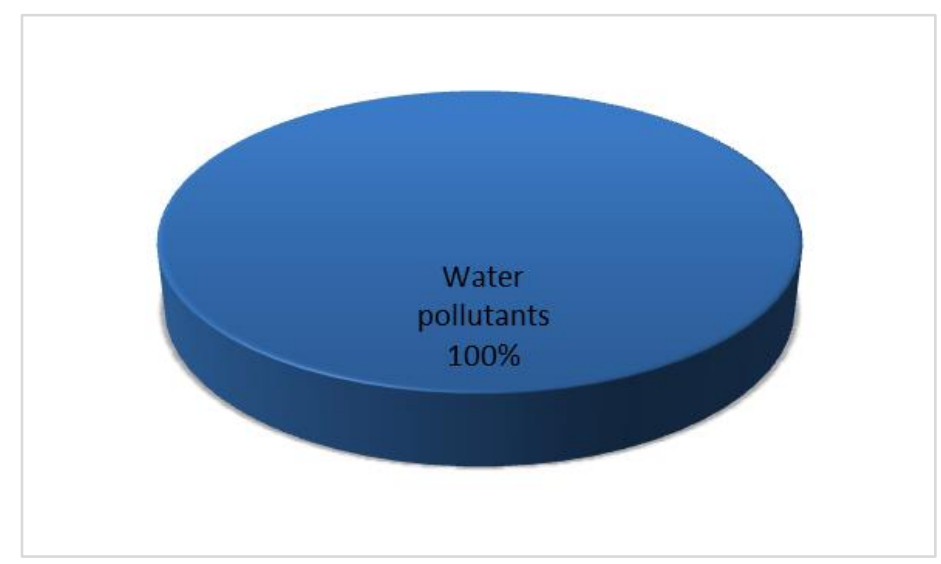

Fig. 7. Percentage of water pollutants at $E_{0}$ 
International Journal of Mathematical, Engineering and Management Sciences

Vol. 3, No. 4, 381-391, 2018

https://dx.doi.org/10.33889/IJMEMS.2018.3.4-027

Fig. 7 shows that water pollutants remain as it is, if soluble and insoluble water pollutants do not exist.

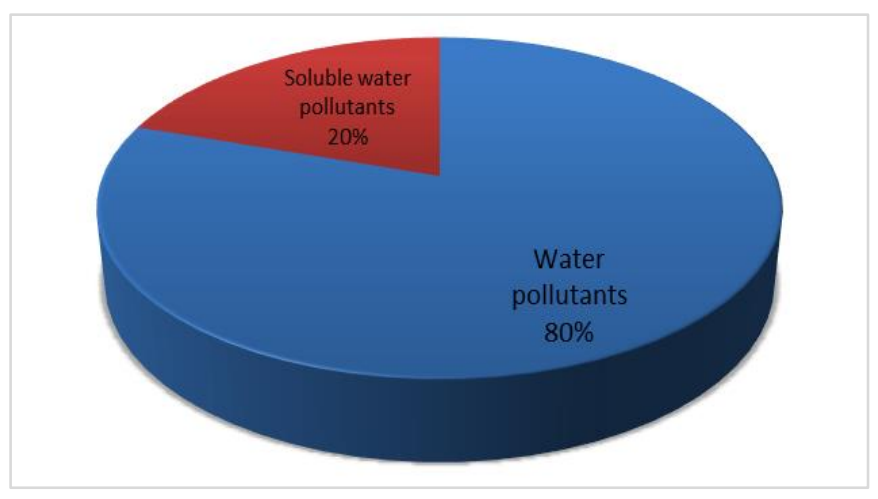

Fig. 8. Percentage of water pollutants at $E_{1}$

Fig. 8 shows that if we do not observe the insoluble water pollutants then $20 \%$ water pollutants are soluble and $80 \%$ water pollutants still exist.

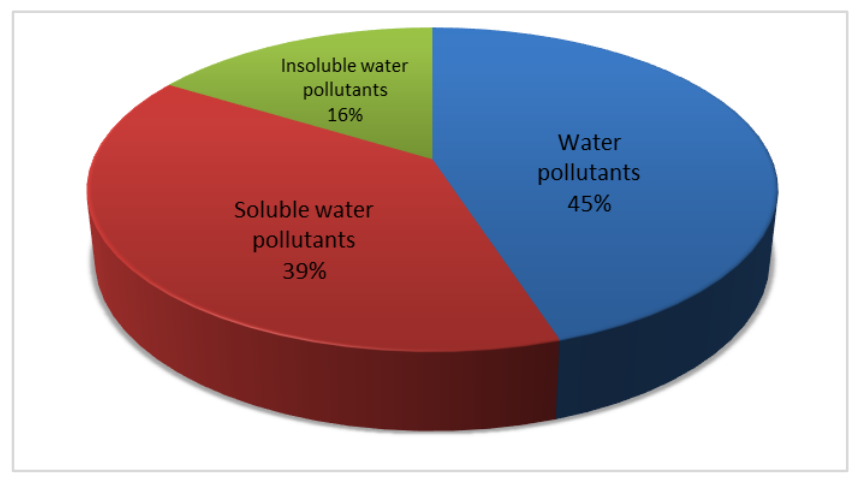

Fig. 9. Percentage of water pollutants at $E^{*}$

Fig. 9 shows that $45 \%$ water pollutants prevail in society, if $16 \%$ water pollutants are insoluble and $39 \%$ are soluble water pollutants.

\section{Conclusions}

Here, a non-linear mathematical model for transmission of water pollutants is formulated. An optimal control is applied to insoluble water pollutants which are converted into soluble water pollutants. The harmful chemicals that come from chemical industries pollute water bodies like streams, ocean, lake, etc. So, we should give proper treatment and try to make them pollution free at some extent. The stability for transmission of water pollutants model is discussed with numerical data. The basic reproduction number is computed as 0.8373 , which shows that control on insoluble water pollutants will be beneficial to reduce the water pollutants by $83.73 \%$. "Water is precious and so it is our responsibility to save". 
International Journal of Mathematical, Engineering and Management Sciences

Vol. 3, No. 4, 381-391, 2018

https://dx.doi.org/10.33889/IJMEMS.2018.3.4-027

\section{Acknowledgements}

The authors will like to thank reviewers for their constructive comments. The authors thank DST-FIST file \# MSI-097 for technical support to the department.

\section{References}

Anderson, R. M. (1991). Discussion: the Kermack-McKendrick epidemic threshold theorem. Bulletin of Mathematical Biology, 53(1-2), 3-32.

Brauer, F. (2008). Compartmental models in epidemiology. In Mathematical Epidemiology (pp. 19-79). Springer, Berlin, Heidelberg.

Diekmann, O., Heesterbeek, J. A. P., \& Roberts, M. G. (2009). The construction of next-generation matrices for compartmental epidemic models. Journal of the Royal Society Interface, 7(47), 873-885.

Ferguson, N. M., Cummings, D. A., Fraser, C., Cajka, J. C., Cooley, P. C., \& Burke, D. S. (2006). Strategies for mitigating an influenza pandemic. Nature, 442(7101), 448-452.

Fleming, W. H., \& Rishel, R. W. (2012). Deterministic and stochastic optimal control (Vol. 1). Springer Science \& Business Media.

Hethcote, H. W. (1994). A thousand and one epidemic models. In Frontiers in mathematical biology (pp. 504-515). Springer, Berlin, Heidelberg.

Pontyiagin, L. S., Boltyanskii, V. G., Gamkrelidze, R. V., \& Mishchenko, E. F. (1986). The mathematical theory of optimal process. Gordon and Breach Science Publishers, NY, USA, 4-5.

Shah, N. H., Satia, M. H., \& Yeolekar, B. M. (2017). Optimum control for spread of pollutants through forest resources. Applied Mathematics, 8, 607-620.

Shah, N. H., Thakkar, F. A., \& Yeolekar, B. M. (2016). Dynamics of parking habits with punishment: An application of SEIR model. Journal of Basic and Applied Research International, 19(3), 168-174.

\section{Links:}

$>$ http://googleweblight.com/i?u=http://www.explainthatstuff.com/waterpollution.html\&hl=en-IN.

$>$ http://googleweblight.com/i?u=http://www.whalefacts.org/water-pollution-facts-causes-effectssolutions/\&hl=en-IN.

$>$ http://www.endmemo.com/chem/insol.php.

$>$ http://www.thunderboltkids.co.za/Grade6/02-matter-and-materials/chapter5.html. 